\title{
Bearing Box Process Specification and Tooling Design
}

\author{
Zhou Cong \\ Zhenjiang Zhongfu Ma Machinery Co., Ltd., Zhenjiang, China \\ Email address: \\ Congzhou2004@126.com
}

\section{To cite this article:}

Zhou Cong. Bearing Box Process Specification and Tooling Design. International Journal of Mechanical Engineering and Applications. Special Issue: Bearing Box Process Specification and Tooling Design. Vol. 8, No. 2, 2020, pp. 65-74. doi: 10.11648/j.ijmea.20200802.12

Received: March 15, 2020; Accepted: March 31, 2020; Published: May 14, 2020

\begin{abstract}
Reasonable process regulations and fixtures can ensure the high efficiency of parts processing and the economy of the enterprise. I started with data collection, then part analysis, then process design, then process specification design, and finally fixture design. In the process design, I obtained the best process route based on the experience of the production site to achieve the effect of saving time and increasing labor productivity. The design of the process specification was based on the production program, the production type was determined, and the process specification was compiled. Production, so the process card of parts and the card of machining process of parts were compiled. The fixture design is to design two sets of tools for the bearing box car mold and the bearing box drilling mold. The bearing box car mold is based on the processed large surface and the two right angle surfaces are used as positioning surfaces. The workpiece is fastened with bolts and nuts. The other large surface is up to size $70, \varphi 180 \mathrm{H} 7$ holes; the bearing box drilling mold is based on the processed large surface, and is positioned at $\varphi 180 \mathrm{H} 7$ holes. The workpiece is fastened with bolts and nuts, and 6- $\varphi 13$ holes are drilled to improve the part. Processing efficiency to ensure the quality of parts processing. In summary, the above-mentioned special fixture design scheme is adopted to ensure the production requirements of parts in batches and improve labor efficiency.
\end{abstract}

Keywords: Bearing Housing, Process Specification, Car Model, Drilling Die, Dimensional Accuracy, Positional Accuracy, Work Efficiency

\section{Part Analysis}

The bearing housing is the main part of the end jumper. The parts are made of HT200 gray cast iron for annealing treatment to ensure the mechanical strength and vibration damping of the parts. The structure of the parts is relatively complicated, so the cast blank is used.

\subsection{Benchmark Selection}

For parts, choose a non-machined surface as a rough reference. For workpieces with several unmachined surfaces, the unmachined surface with higher relative positional accuracy with the machining requirements should be used as the reference rough reference. The selection of the fine benchmark should mainly consider the problem of benchmark overlap. When the design basis and the process benchmark do not coincide, the size conversion should be performed [1]. When the part is machined, the machined large surface is used as the reference, and the two right angle surfaces are used as the positioning surface: the large surface limits 3 degrees of freedom, and the two right angle surfaces limit 3 degrees of freedom. When drilling the part, the part is positioned with $\varphi 180 \mathrm{H} 7$ hole as the reference, and the two right-angled faces are used as the positioning surface: the large surface limits 3 degrees of freedom, the $\varphi 180 \mathrm{H} 7$ hole limits 2 degrees of freedom, and the right-angled surface limits 1 Degree of freedom.

\subsection{Determine the Type of Production}

The production program of the known parts is 120 pieces/year. Check the "Machinery Manufacturing Process and Equipment Design Manual" to determine that these parts are in the middle batch production.

\subsection{Process Design Basis}

Since the workpiece has an annual output of 120 pieces, it is a medium-volume production. Due to the complicated structure of the part, high dimensional accuracy and geometrical tolerance requirements, it is difficult to guarantee the dimensional accuracy and positional accuracy by ordinary 
processing methods, which is time consuming and laborious, the processing efficiency is low, the processing quality is difficult to guarantee, and the scrap rate is high (parts are shown in the figure 1). Therefore, it is necessary to design the

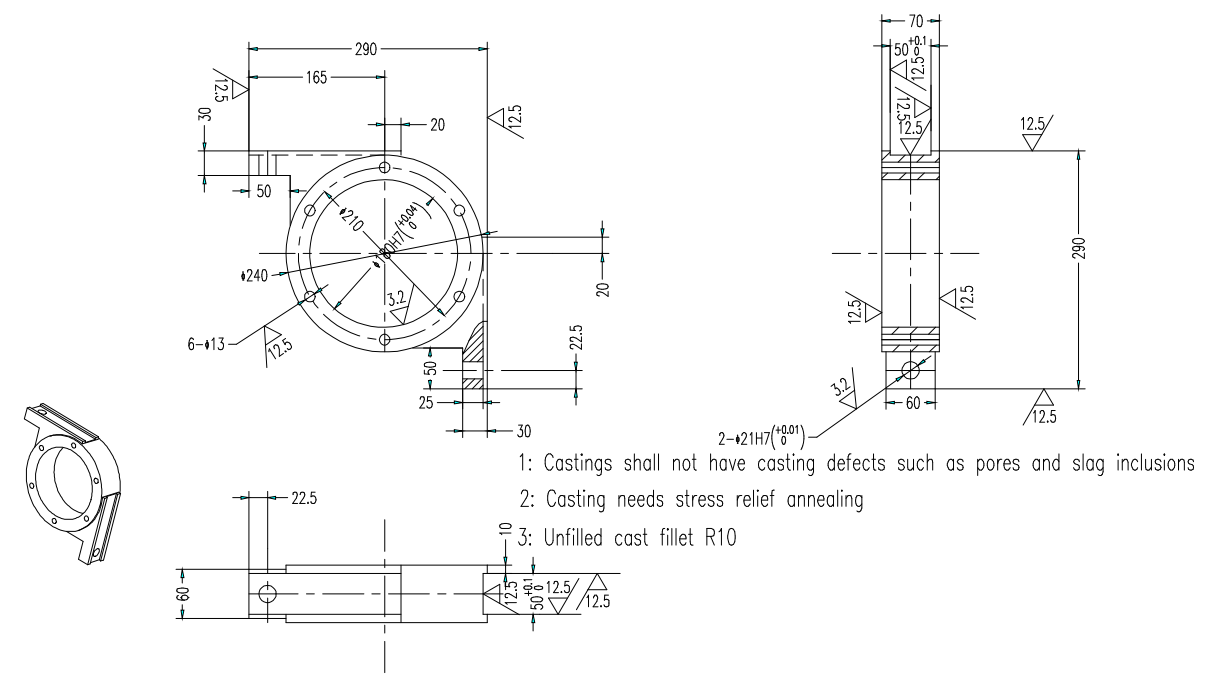

Figure 1. Bearing housing.

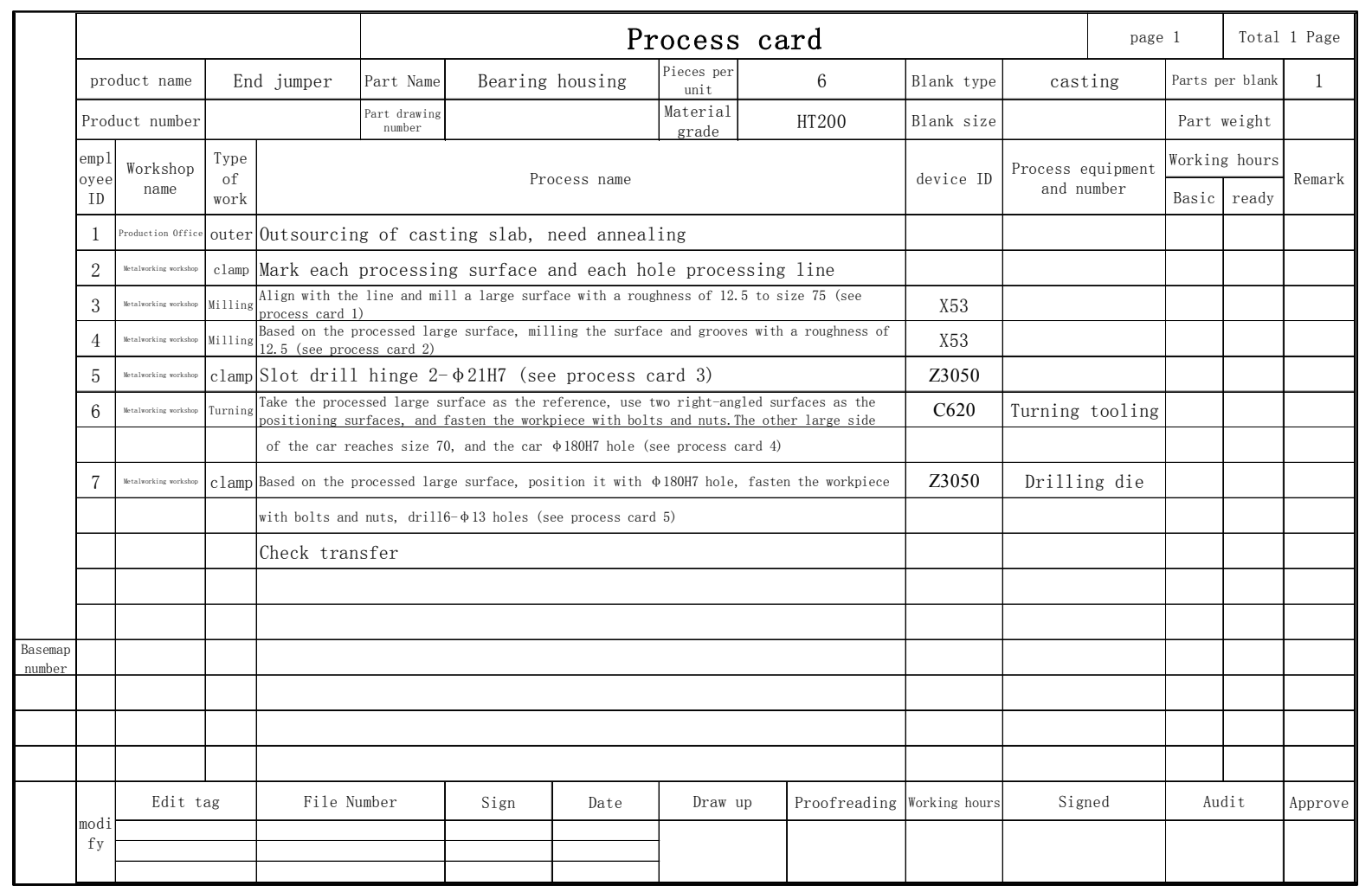

Figure 2. Parts Process Card.

\section{Process Design}

\subsection{Part Process Card Design (See Figure 2)}

Process 1: slab blank, need to be annealed

Step 2: Draw each processing surface and hole processing line

Step 3: Align the line and mill a large surface with a process specification for the part, compile the part process card, draw the part blank drawing, compile the part machining process card, and design the part processing die and the drilling die [2] to improve the work efficiency. 
right-angled surfaces are used as the positioning surface, and the workpiece is fastened with bolts and nuts. The other large surface of the car reaches the size of 70 , and the car is $\varphi 180 \mathrm{H} 7$ (see the process card 4) (C620 lying Lathe)

\subsection{Part Blank Design (See Figure 3)}

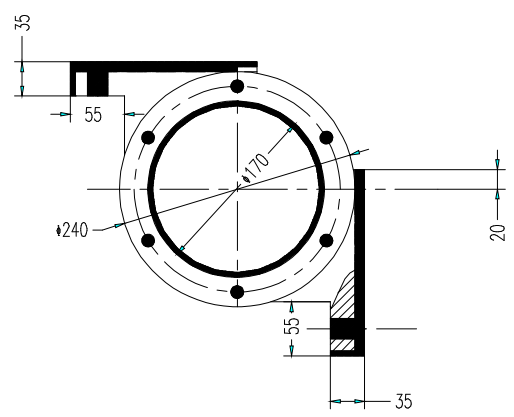

Step 7: Position the $\varphi 180 \mathrm{H} 7$ hole on the basis of the processed large surface, and fasten the workpiece with bolts and nuts, and drill 6- $\varphi 13$ holes (see process card 5) (Z3050 radial drilling machine)

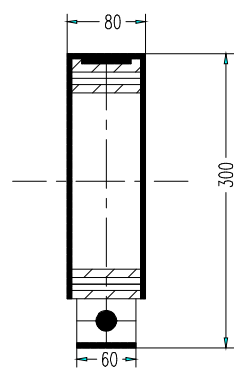

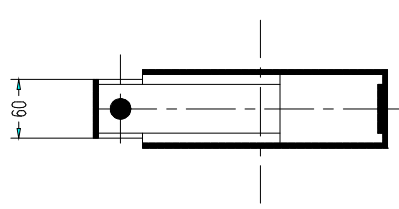

Figure 3. Part blank drawing.

\subsection{Part Process Card Design}

\subsubsection{Process Card 1 (See Figure 4)}

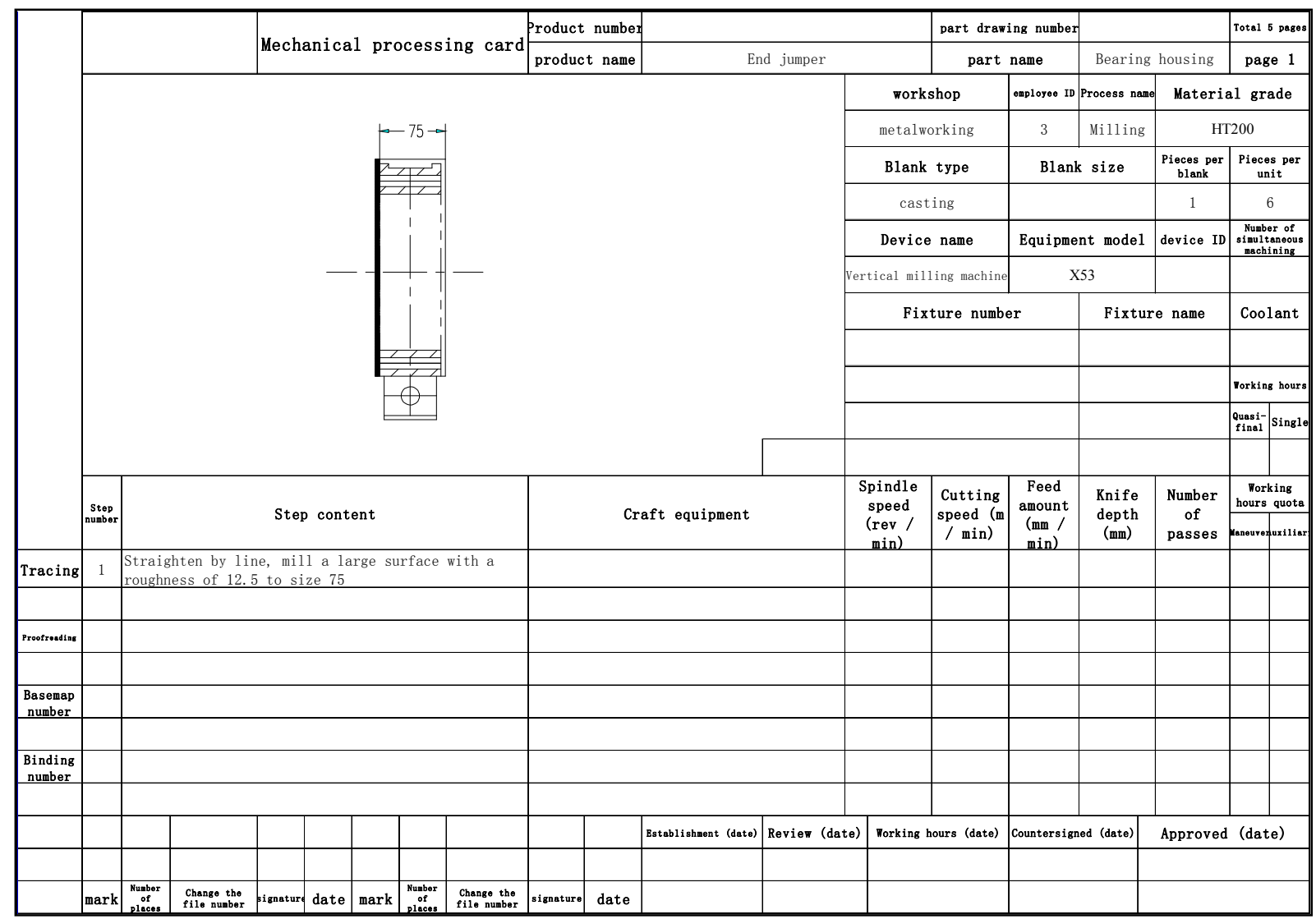

Figure 4. Parts Process Card 1. 


\subsubsection{Process Card 2 (See Figure 5)}

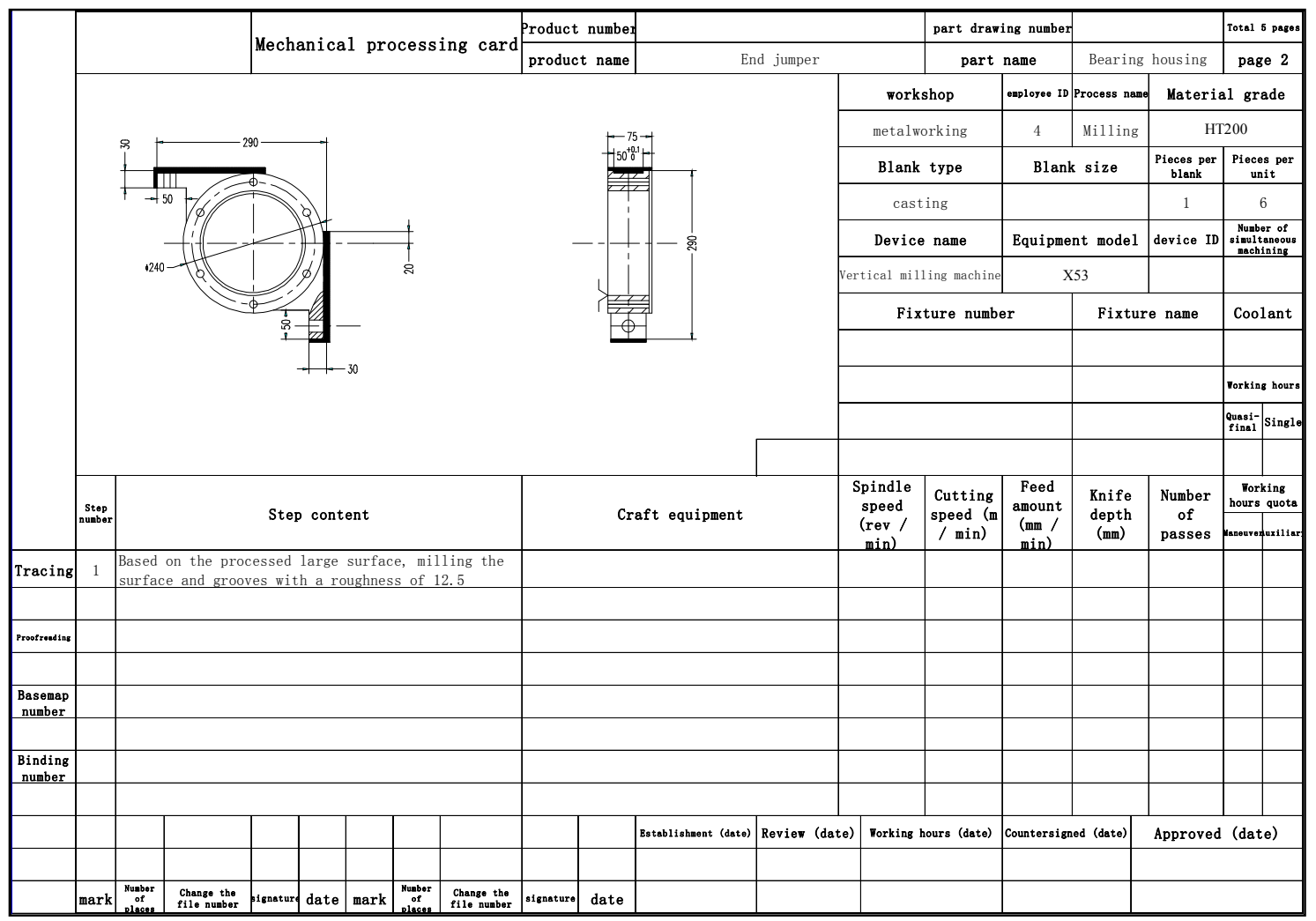

Figure 5. Parts Process Card 2.

\subsubsection{Process Card 3 (See Figure 6)}

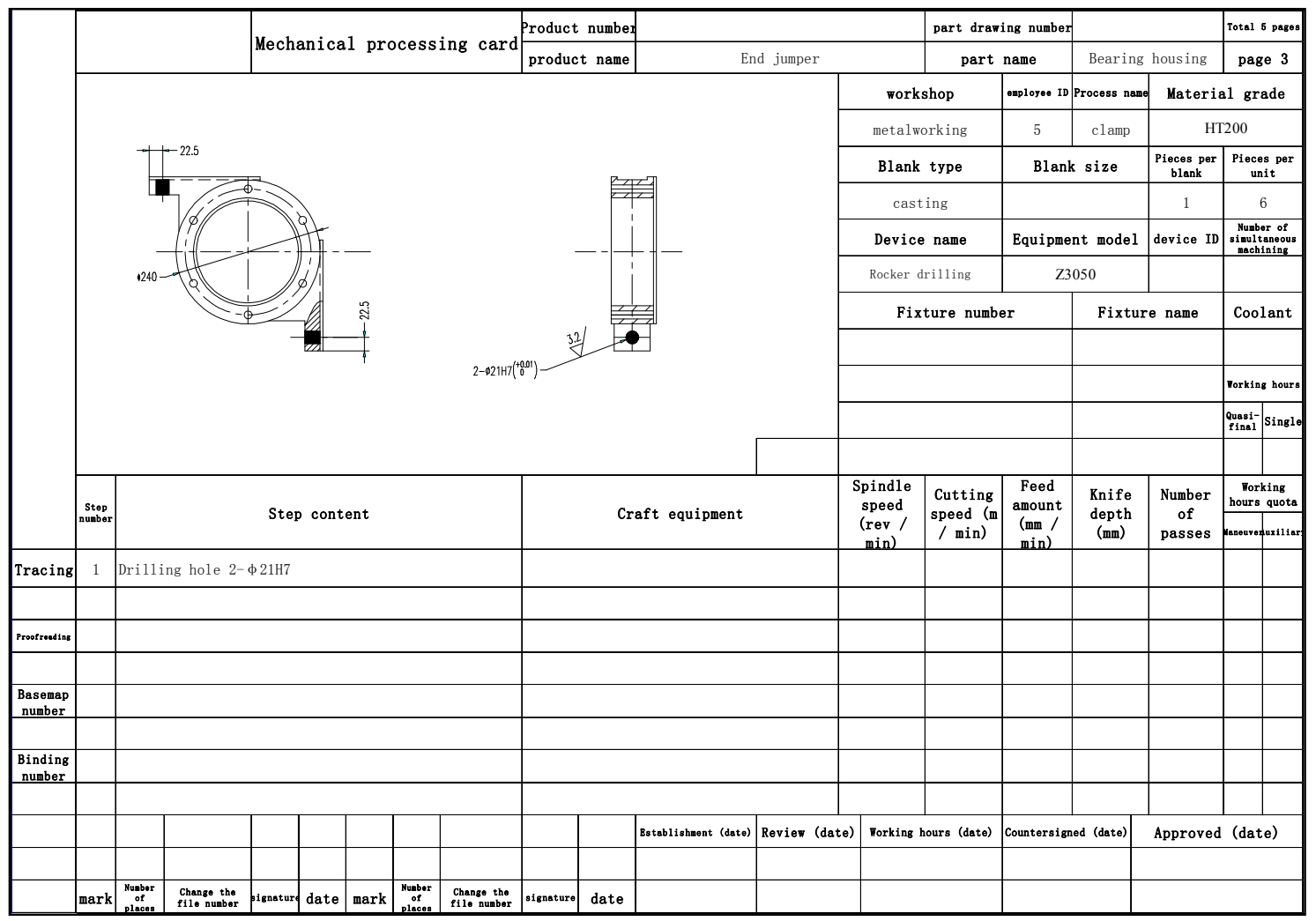

Figure 6. Parts Process Card 3. 


\subsubsection{Process Card 4 (See Figure 7)}

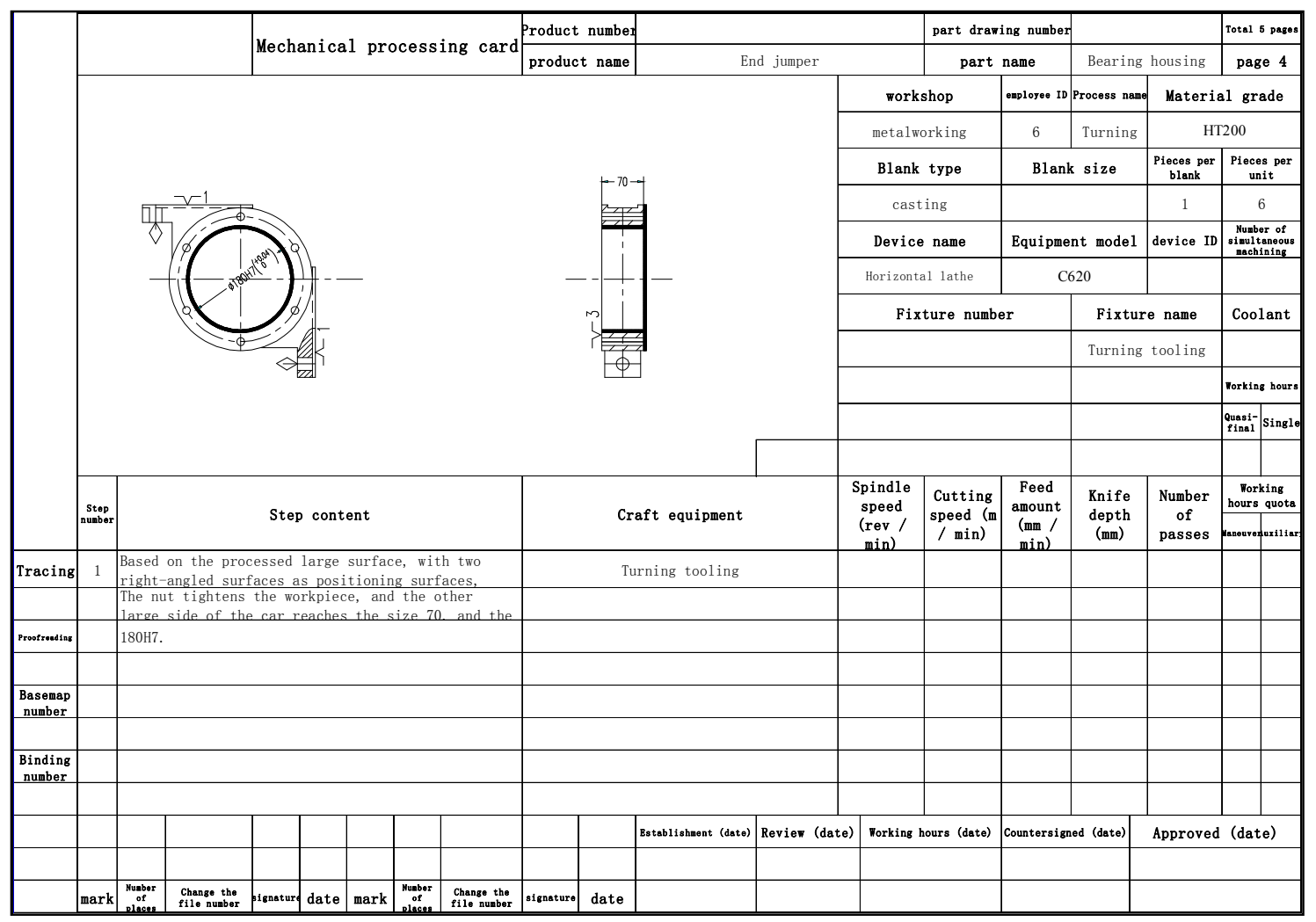

Figure 7. Parts Process Card 4.

\subsubsection{Process Card 5 (See Figure 8)}

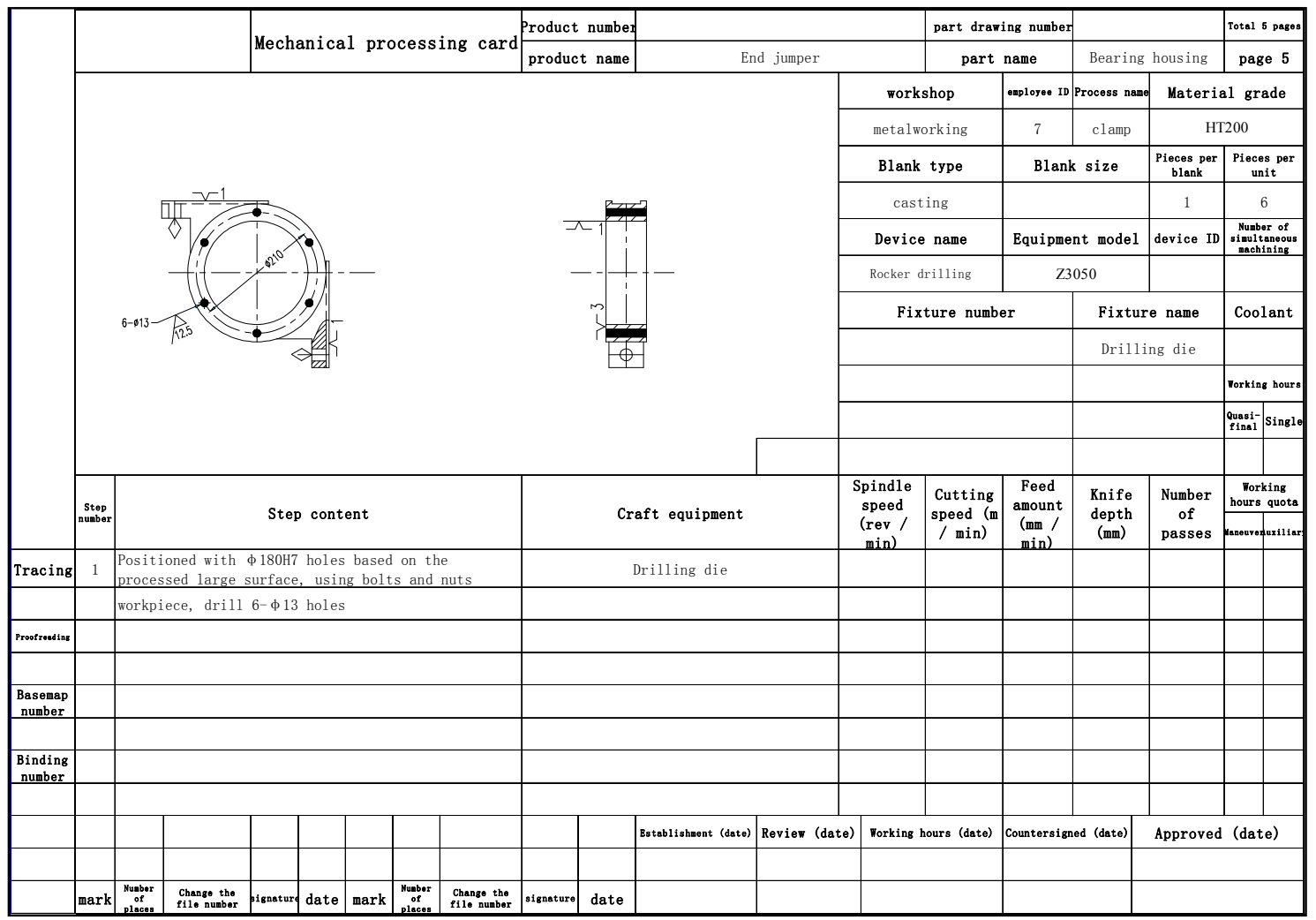

Figure 8. Parts Process Card 5. 


\section{Fixture Design}

\subsection{Bearing Housing Model}

The bearing housing has a complicated structure and an irregular shape. It is impossible to realize the machining of the hole and its end face without special tooling. For this reason, in the design of the tooling, the large surface of the workpiece has been used as the positioning reference, and the two right-angled surfaces of the workpiece are used as the positioning surface. The workpiece is fastened with bolts and nuts, and the auxiliary support is used to clamp the workpiece with the ejector pin. The large face is 70 in size and the car is $\varphi 180 \mathrm{H} 7$ [3].

\subsubsection{Bearing Box Body Tooling Structure}

The bearing box body tooling structure is shown in Figure 9. The bearing box body tooling is mainly composed of bottom plate, support plate, auxiliary support, ejector pin, support, bolt M20×90, nut M20, screw M20×100, bolt M10×60., nut M8, handle M8 and so on.
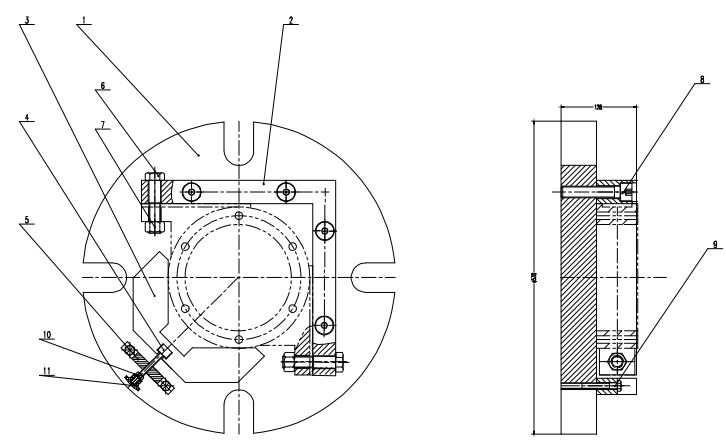

Figure 9. Bearing housing model.

1: Base plate 2: Support plate 3: Auxiliary support 4: Plunger 5: Support 6: Bolt M20×90

7: Nut M20 8: Screw M20×100 9: Bolt M10×60 10: Nut M8 11: Handle M8

\subsubsection{Working Principle of Bearing Box Body Tooling}

Place the bearing housing model on the four-jaw chuck of the C630 horizontal lathe, so that the end surface of the bottom plate fits on the end surface of the claw, adjust the claw to initially clamp the bottom plate of the tooling, and then use the table to find the end surface and outer circle of the bottom plate of the tooling, and then Clamp the bottom plate with four claws. The large surface of the workpiece has been machined on the bottom plate of the tooling, and the two right-angled faces of the workpiece are used as the positioning surface. The workpiece is fastened with bolts and nuts, and the auxiliary support is used to clamp the workpiece with the ejector pin. 70, car $\varphi 180 \mathrm{H} 7$ hole [4].

By using the self-designed bearing housing model, the other large surface of the bearing housing and the $\varphi 180 \mathrm{H} 7$ hole can meet the dimensional accuracy requirements of the drawings, ensure the consistency of mass production, solve the problem of low processing efficiency, and improve Work efficiency and various performance indicators [5].

\subsubsection{Main Parts Design of Bearing Box Body Tooling}

\section{i. Backplane}

The design of the bottom plate is shown in Figure 10. The part is connected with the workpiece, the support plate, the auxiliary support, the support, the screw and the bolt, and is the basic part of the tooling. The bottom plate material selects 45\# medium carbon quenched and tempered structural steel, which has high strength and good machinability after quenching and tempering treatment, and has certain toughness, plasticity And wear resistance [6].
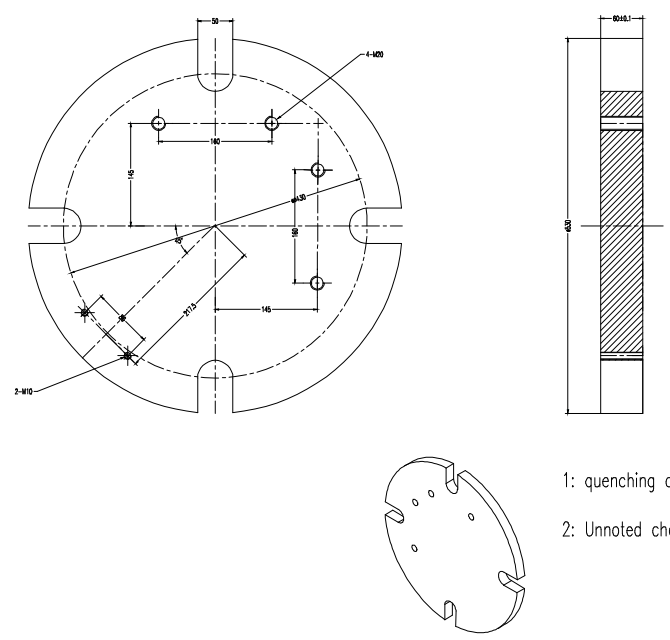

1: quenching and tempering HB217-255 2: Unnoted chamfer $\mathrm{Cl}$

Figure 10. Bottom plate.

\section{ii. Support Plate}

The design of the support plate is shown in Figure 11. The part is connected to the workpiece, the bottom plate, the bolt, the nut and the screw for positioning and clamping of the workpiece. The material of the support plate is $45 \#$ medium carbon quenched and tempered structural steel. After quenching and tempering treatment, it has high strength and good machinability, and has certain toughness, plasticity and wear resistance [7].

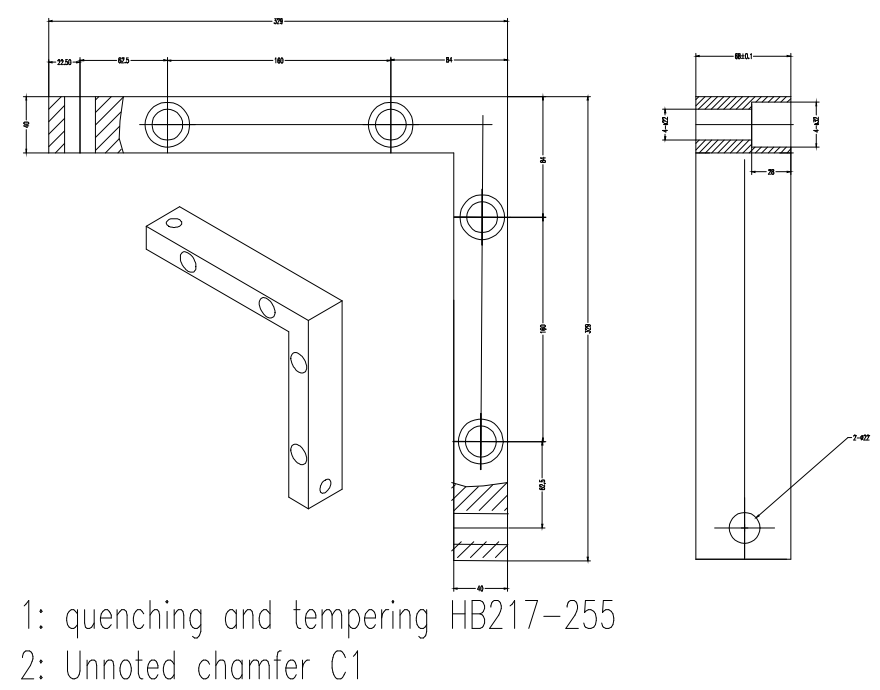

Figure 11. Support plate. 


\section{iii. Auxiliary Support}

The design of the auxiliary support is shown in Figure 12. The part is used for auxiliary positioning of the workpiece and is connected to the ram, the bottom plate and the workpiece. The auxiliary support material selects $45 \#$ medium carbon quenched and tempered structural steel, which has high strength and good machinability after quenching and tempering treatment, and has certain toughness, plasticity and wear resistance.

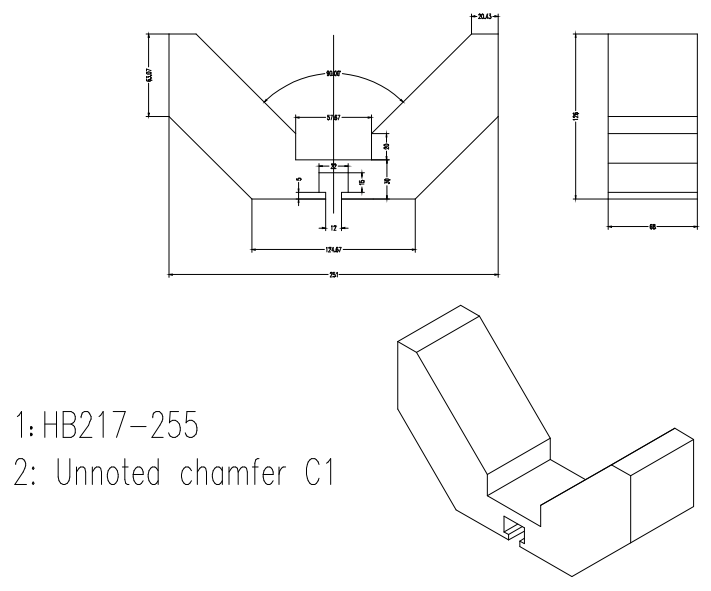

Figure 12. Auxiliary support.

\section{iv. Ejector}

The design of the ram is shown in Figure 13, which is connected to the support, the auxiliary support, the handle, and the nut. The top rod material selects $45 \#$ medium carbon quenched and tempered structural steel, which has high strength and good machinability after quenching and tempering treatment, and has certain toughness, plasticity and wear resistance.
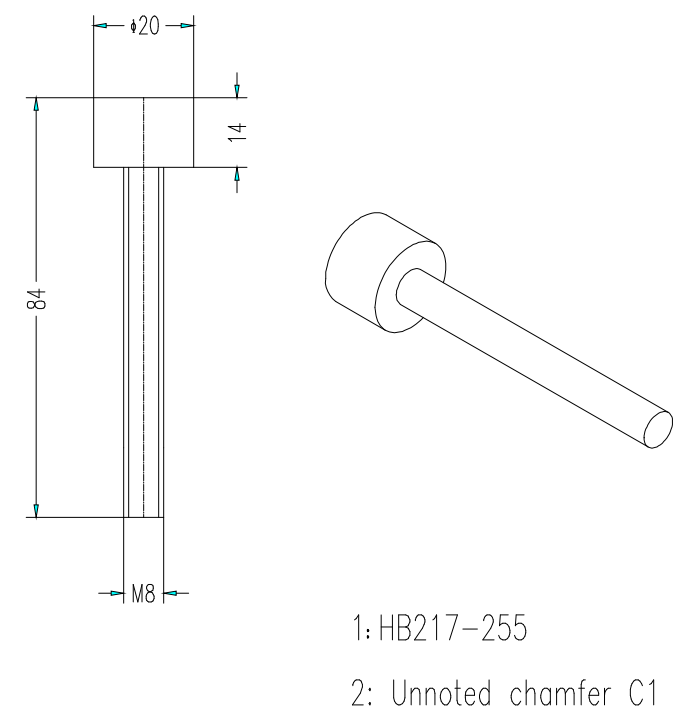

Figure 13. Ejector.

\section{v. Support}

The design of the support is shown in Figure 14. The part is connected to the bottom plate, the ejector pin and the bolt, and is mainly used for auxiliary positioning of the workpiece. The bearing material selects $45 \#$ medium carbon quenched and tempered structural steel, which has high strength and good machinability after quenching and tempering treatment, and has certain toughness, plasticity and wear resistance
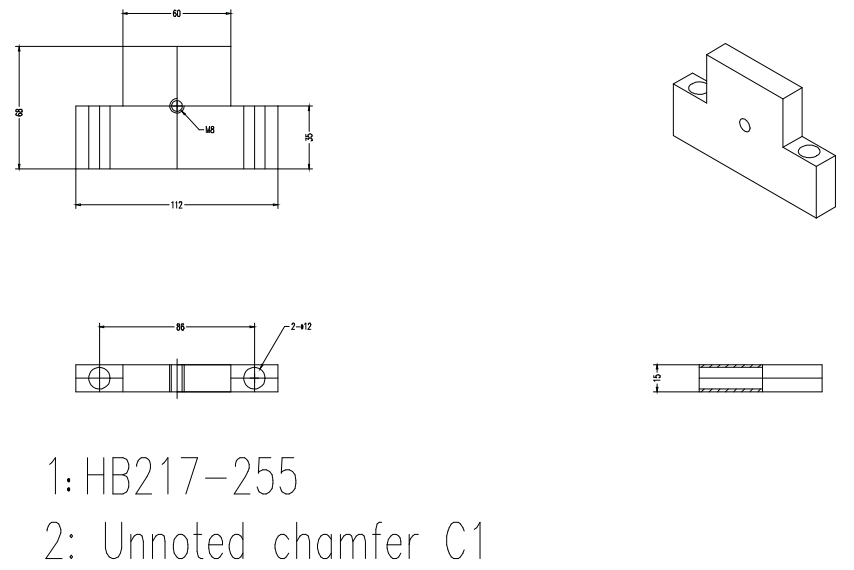

Figure 14. Support.

\subsection{Bearing Housing Drilling Mold}

There are $6-\varphi 13$ holes on the large surface of the bearing housing. If the scribing is used, the machining efficiency is very low, and the machining accuracy is difficult to guarantee. Therefore, it is considered to design a large surface, a hole and a right angle as the positioning reference. The die-casting machine has $6-\varphi 13$ holes on the large surface of the bearing housing.

\subsubsection{Bearing Box Body Drilling Tooling Structure}

The bearing box body drilling tooling [8] structure is shown in Figure 15: The bearing box body drilling tooling mainly consists of the bottom plate, the support, the drill template, the hinge pin, the pressure plate, the contour pin 1, the drill sleeve $\varphi 13 \times 20 \times 40$, the stud M20 $\times 125$, nut M20, screw M20 $\times 100$, cotter pin $\varphi 10 \times 45$, stud M20 $\times 150$, contour pin 2 , etc.

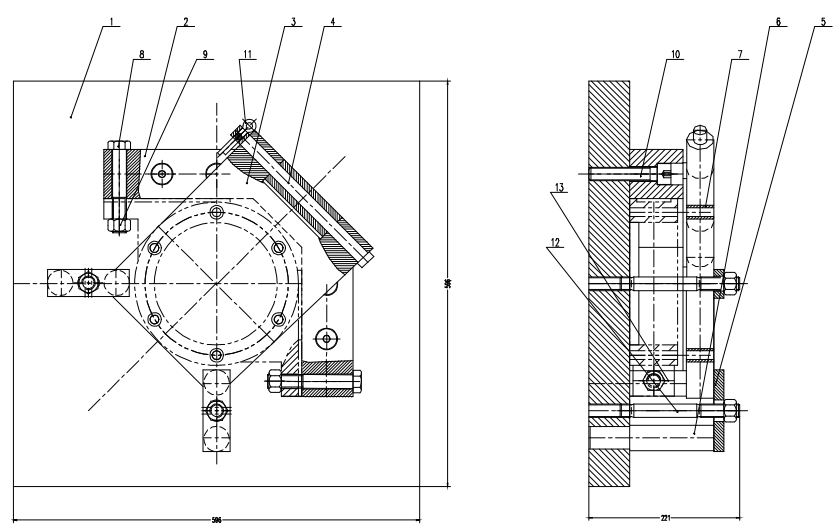

Figure 15. Bearing housing mold.

1: Base plate 2: Support 3: Drill template 4: Hinge pin 5: Press plate 6: Contour pin 1 7: drill sleeve $\varphi 13 \times 20 \times 40$ 8: stud M20×125 9: nut M20 10: screw M20×100 11: Cotter pin $\varphi 10 \times 45$ 12: Stud M20×150 13: Contour pin 2 


\subsubsection{Working Principle of Bearing Box Drilling Tooling}

Place the bearing box drill on the Z3050 radial drilling machine [9] table, press the bottom plate with the pressure plate, clamp the large-faced template of the workpiece, and drill 6- $\varphi 13$ holes through the drill sleeve [10].

By using the self-designed bearing housing body to mold the bearing housing 6- $\varphi 13$ hole, to meet the dimensional accuracy requirements of the drawings, to ensure the consistency of mass production, solve the problem of low processing efficiency, improve the ergonomics And various performance indicators.

\subsubsection{Main Parts Design of Bearing box Drilling Tooling}

\section{i. Backplane}

The design of the bottom plate is shown in Figure 16. The part is connected with the workpiece, the support, the contour pin 1 , the contour pin 2 , the screw and the stud, and is the basic component of the tooling. Since the contour pin $\varphi 33 \mathrm{k} 6$ is a positive tolerance, the cooperation of the bottom plate and the contour pin is an interference fit. Since the surface roughness of the $4-\varphi 33 \mathrm{H} 7$ holes is 3.2 , the process arrangement requires reaming [11]. The material of the bottom plate is selected from $45 \#$ medium carbon quenched and tempered structural steel [12], which has high strength and good machinability after quenching and tempering treatment, and has certain toughness, plasticity and wear resistance.
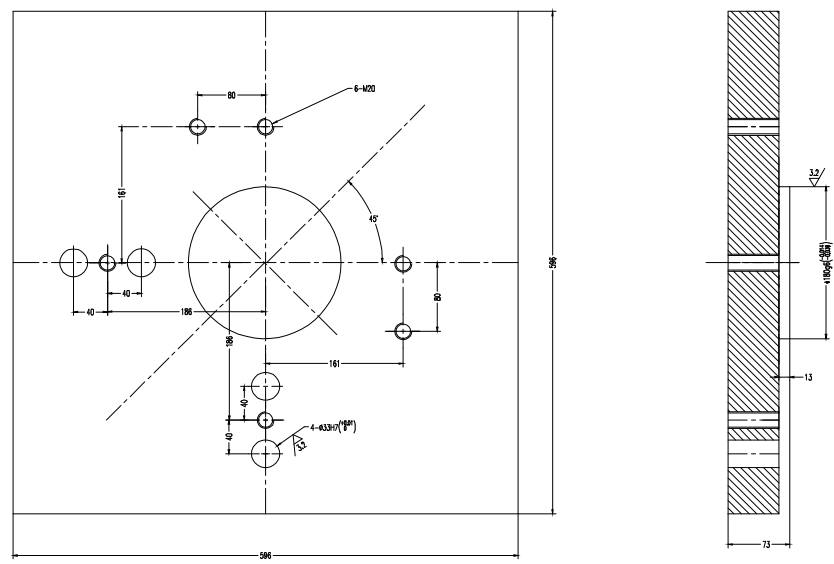

Figure 16. Bottom plate.

\section{ii. Support}

The design of the support is shown in Figure 17. The parts are connected to the workpiece, the bottom plate, the drill template, the hinge pin, the bolt, the nut, the screw [13], and the like. There are $\varphi 20 \mathrm{H} 7$ holes in the design of the support. Since the outer precision of the hinge pin is negative tolerance, the cooperation between the support and the hinge pin is a clearance fit. The surface roughness of the $\varphi 20 \mathrm{H} 7$ hole is 3.2, and the process needs to arrange fine boring. The HT200 gray cast iron parts are selected for annealing treatment, and the blanks are required to have no casting defects such as porosity and shrinkage to ensure the bearing has certain mechanical strength and vibration damping.

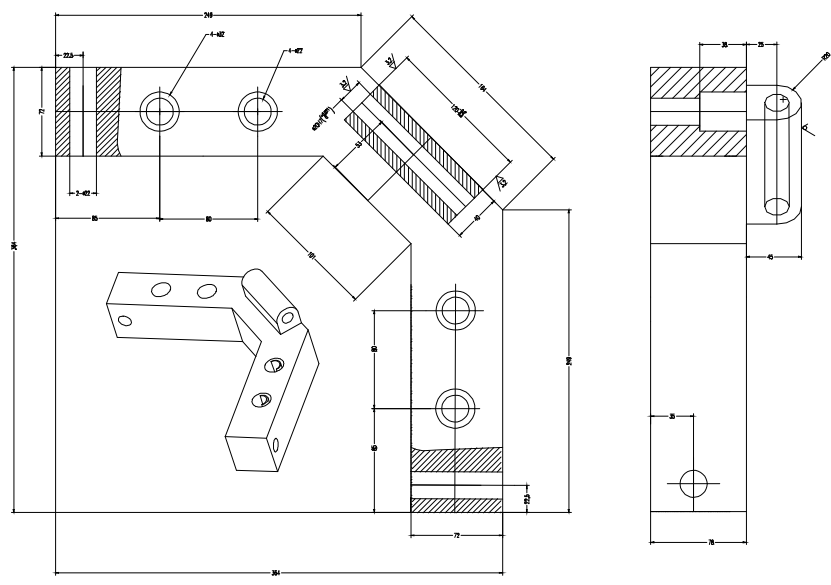

Figure 17. Support.

\section{iii. Drill Template}

The design of the drill template [14] is shown in Figure 18, which is connected to the support, the hinge pin, the pressure plate, and the drill sleeve. Since the hinge pin $\varphi 20 \mathrm{~g} 6$ is a negative tolerance, the fit of the drill template and the hinge pin is a clearance fit to facilitate the rotation of the drill template; since the $6-\varphi 20 \mathrm{H} 7$ hole is connected to the drill sleeve, and the outer tolerance of the drill sleeve is a positive tolerance, the drill template is drilled. The fit with the drill sleeve is an interference fit. Since the surface roughness of the $\varphi 20 \mathrm{H} 7$ and $6-\varphi 20 \mathrm{H} 7$ holes is 3.2 , the process arrangement requires reaming or fine boring. The drilling template material selects $45 \#$ medium carbon quenched and tempered structural steel, which has high strength and good machinability after quenching and tempering treatment, and has certain toughness, plasticity and wear resistance.
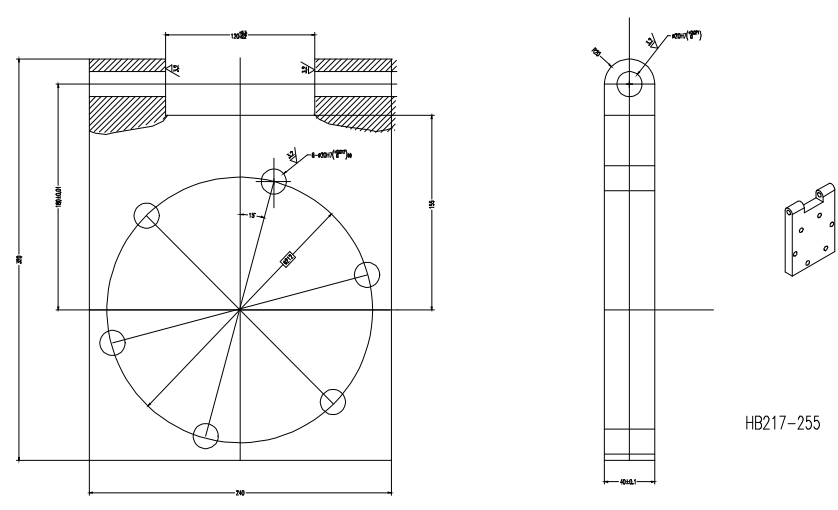

Figure 18. Drill Template.

\section{iv. Hinge Pin}

The design of the hinge pin [15] is shown in Figure19, which is connected to the support, the drill template, and the split pin. The hinge pin is designed with a tolerance of $\varphi 20 \mathrm{~g} 6$ outer circle. The size of the support and the drill template hole is $\varphi 20 \mathrm{H} 7$, so the fit of the hinge pin to the support and the drill template is clearance fit. The surface roughness of $\varphi 20 \mathrm{~g} 6$ is 3.2, and the process needs to be ground. The hinge pin material selects $45 \#$ medium carbon quenched and tempered structural steel, which has high strength and good machinability after quenching and tempering treatment, and 
has certain toughness, plasticity and wear resistance.
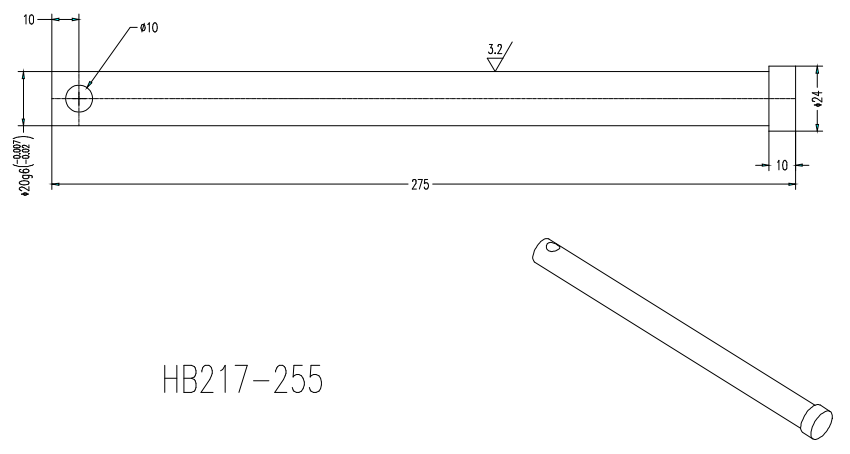

Figure 19. Hinge pin

\section{v. Platen}

The design of the pressure plate is shown in Figure 20. This part is used for the compression of the drill template, and is connected to the drill template, the contour pin and the nut. The pressure plate material selects Q235-A ordinary carbon steel, which is economical.
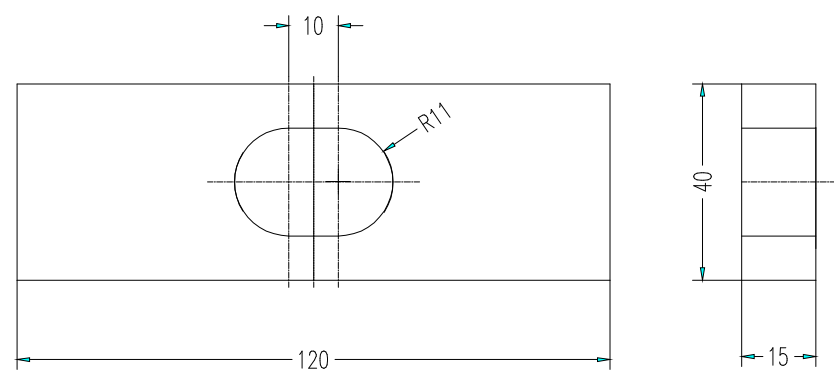

Figure 20. Pressure plate.
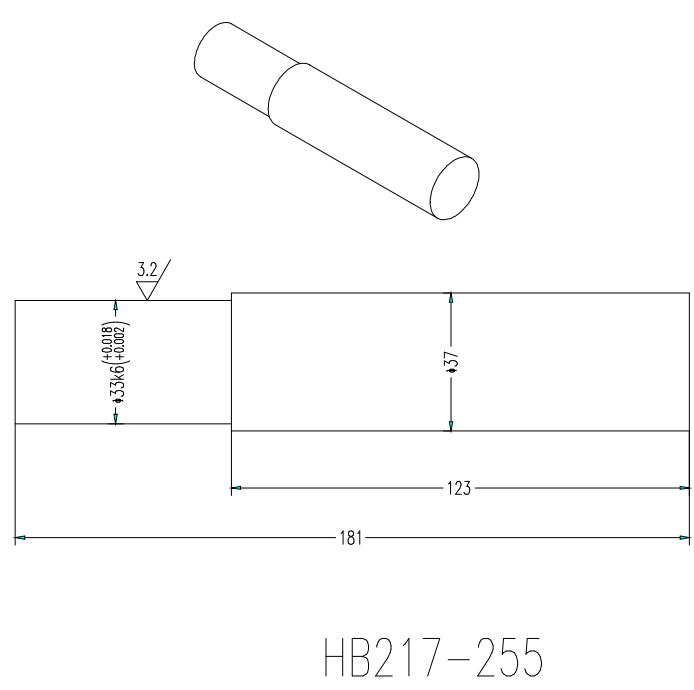

Figure 21. contour pin 1.

\section{vi. Contour Sales 1}

The design of the contour pin 1 is shown in Figure 21. The part is a transitional part connected to the bottom plate and the pressure plate. The contour pin design is marked with a $\varphi 33 \mathrm{k} 6$ outer circle tolerance, and the bottom plate $\varphi 33 \mathrm{H} 7$ hole is matched with an interference fit. The surface roughness is 3.2 , so the process arrangement needs to be ground. The contour pin 1 material selects $45 \#$ medium carbon quenched and tempered structural steel, which has high strength and good machinability after quenching and tempering treatment, and has certain toughness, plasticity and wear resistance.
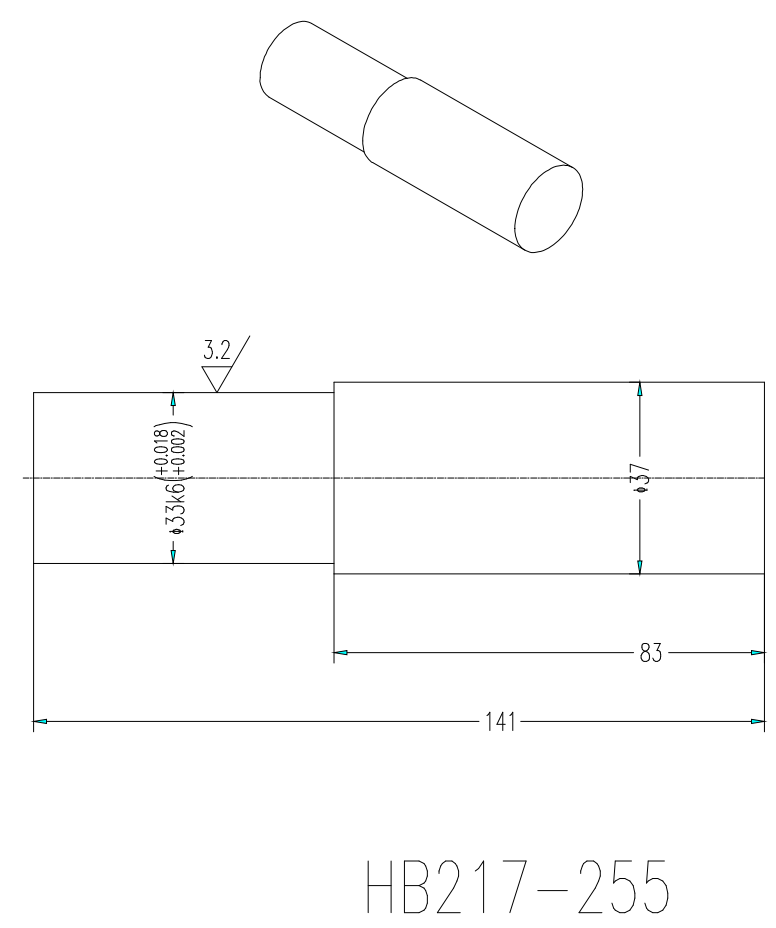

Figure 22. Contour pin 2.

\section{vii. Contour 2}

The design of the contour pin 2 is shown in Figure 22. The part is a transitional part connected to the bottom plate and the drill template. The contour pin design is marked with a $\varphi 33 \mathrm{k} 6$ outer circle tolerance, and the bottom plate $\varphi 33 \mathrm{H} 7$ hole is matched with an interference fit. The surface roughness is 3.2 , so the process arrangement needs to be ground. Contoured pin 2 material selects $45 \#$ medium carbon quenched and tempered structural steel, which has high strength and good machinability after quenching and tempering treatment, and has certain toughness, plasticity and wear resistance.

\section{The Conclusion}

After the preparation of the process specification and the installation of the fixtures, the machining accuracy of the bearing housing is improved, the problem of machining and drilling of the bearing housing is solved, the processing load of the operator is reduced, the labor intensity is reduced, and the work is shortened. The production cycle greatly increases productivity and guarantees product quality.

\section{References}

[1] Wang Shaojun. Mechanical Manufacturing Process Design Handbook. Harbin Industrial Press. 
[2] Shanghai Diesel Engine Process Equipment Research Institute. Metal Cutting Machine Tool Fixture Design Manual. Mechanical Industry Press.

[3] Shanghai Science and Technology Press, Northeast Heavy Machinery Institute, Luoyang Branch. Machine Tool Fixture Design Manual.

[4] Shen Xueqin. Tolerance coordination technology measurement. Higher Education Press.

[5] Wu Zongze, Luo Shengguo. Mechanical Design Course Design Manual. Higher Education Press.

[6] Zhang Jie, Li Xianmin, Zhao Hu. Technical basis of mechanical manufacturing. Southwest Jiaotong University Press.

[7] Liu Hongwen. Materials Mechanics. Higher Education Press.

[8] Huang Heting, Wang Furong, Liu Jianming. Mechanical Manufacturing Equipment. Mechanical Industry Press.
[9] Qiu Xuanhuai. Mechanical Design. Higher Education Press

[10] Li Yimin. Concise manual for mechanical manufacturing process design. Mechanical Industry Press.

[11] Ai Xing, Xiao Shigang. Concise Handbook of Cutting Amount. Mechanical Industry Press.

[12] Design Manual for Mechanical Engineers. Chemical Industry Press.

[13] Sui Hongjun Wu Runyan. The latest practical hardware manual. Henan Science and Technology Press.

[14] Lv Yingbo. Manual of Mechanical Drawing. Chemical Industry Press.

[15] Zhou Kaiqin. Manual of Mechanical Parts. Higher Education Press. 